\title{
Die Mira Lobe Schule Hannover - Inklusion und BNE unter einem Dach
}

\section{Zum Hintergrund}

Die Mira Lobe Grund-, Förder- und Oberschule in Trägerschaft der DIAKOVERE Annastift Leben und Lernen gGmbH ist eine staatlich anerkannte Ersatzschule in Hannover-Mittelfeld. Hervorgegangen aus einer Förderschule für körperliche und motorische Entwicklung, steht sie seit einigen Jahren auch Kindern und Jugendlichen ohne Handicap offen. Sie bietet allen ein breites Spektrum an vielfältigen Lernangeboten und Entwicklungsmöglichkeiten. Die enge Verzahnung der drei Schulzweige ermöglicht ein kompetenzorientiertes pädagogisches Angebot in multiprofessionellen Teams. Lehrkräfte für Grund-, Förder- und Oberschulen, pädagogische Mitarbeitende in unterrichtsbegleitender Funktion sowie pädagogische Mitarbeitende in therapeutischer Funktion arbeiten Hand in Hand. Es wird von den Stärken der einzelnen Kinder ausgegangen. In einem gemeinschaftlichen Rahmen wird ihnen eigenverantwortliches Lernen ermöglicht. Die Verschiedenheit aller Kinder und Jugendlichen wird als Gewinn erlebt.

\section{Die Umsetzung}

Tatsächlich steht die Kombination von Bildung für nachhaltige Entwicklung (BNE) und Inklusion in der Mira Lobe Schule noch ganz am Anfang. Auch nach fast acht Jahren inklusiver Erfahrung ist man noch auf dem Weg und vielfach auf der Suche nach Möglichkeiten der Umsetzung im (gemeinsamen) Schulalltag.

Eine Vielzahl der 17 UN-Nachhaltigkeitsziele (Sustainable Development Goals, SDGs) werden bisher regelmäßig berücksichtigt. Das drückt sich z. B. folgendermaßen aus: Am Rande des Schulhofs gibt es seit einiger Zeit eine große Bienenweide. Zukünftig wird die Schule eigene Bienen bekommen. Außerdem werden in das während einer Projektwoche umgebaute Tierhaus mit Außenbereich Hühner einziehen. Der Schulgarten wirft so manches ab, was vom Praxistag „Gartenbau“" oder einzelnen Klassen verarbeitet oder verkauft wird. Ein Aquarium mit zahlreichen bunten Fischen sowie ein Terrarium mit Schrecken werden gepflegt. Lange wurden fleißig Flaschendeckel aus Plastik für den Verein „Assistenzhunde Deutschland e.V." gesammelt. Das durch Weiterverkauf an eine Recyclingfirma eingenommene Geld wurde für die Ausbildung von Assistenzhunden verwendet. Der Praxistag Cafeteria versucht Müll zu vermeiden (wiederverwendbares Geschirr, keine Trinkhalme aus Plastik, Pfandflaschen). Was einzelne Klassen unternehmen, um Nachhaltigkeit zu fördern, wird durch die vielfältigen Antworten auf entsprechende Interviewfragen deutlich, die den Jugendlichen in den Jahrgängen 7 und 8 kürzlich im Auftrag von Frau Prof. Vierbuchen und Herrn Prof. Rieckmann (Universität Vechta) gestellt wurden (zusammengefasste Ergebnisse s. u.).

Durch die gefestigte inklusive Beschulung werden in der Mira Lobe Schule weitere Themen berührt, die über den Umweltschutz hinausgehen, aber Teil der SDGs sind: Es geht vor allem um Gesundheits- und Bewegungsförderung. Zahlreiche Sportprojekte sowie ein Schulzirkus, Bewegungs- und Fitnessgruppen wurden ins Leben gerufen. Die Schule nimmt am Schulobstprogramm des Landes Niedersachsen teil, bietet Sportfahrten an (Ski, Kanu, Segeln und Radfahren) und kooperiert mit verschiedenen Sportvereinen. Angebote werden so gestaltet, dass alle Schülerinnen und Schüler daran mitwirken können, ob mit oder ohne Behinderung. Regelmäßig nehmen Schülerinnen und Schüler der Mira Lobe Schule an Wettkämpfen im Rahmen von „Jugend trainiert für Paralympics“ teil, oftmals sehr erfolgreich. Im Sommer 2019 ist der Schule das Siegel „Sportfreundliche Schule“ verliehen worden.

Ein besonderes inklusives Setting, wie man es in der Mira Lobe Schule findet, verlangt nach einem besonderen Unterricht: Offene (Lern-)Systeme sollen den Schülerinnen und Schülern Selbstvertrauen in ihre eigenen Fähigkeiten vermitteln. Individuelle Lerntempi, speziell angepasste Programme, Zeugnisse in Notenform erst ab Klasse 9, eine gründliche $\mathrm{Zu}$ kunftsplanung und vieles mehr gehören deshalb unabdingbar dazu. Denn jedes Kind ist anders und lernt auf seine eigene Art und Weise. Es wird angestrebt, alle Schülerinnen und Schüler mit dem größtmöglichen Wissen (im praktischen, im theoretischen, aber auch im sozialen Bereich) aus der Schule zu entlassen. An der Mira Lobe Schule werden alle Abschlüsse vergeben, die man im Sekundarbereich I erwerben kann.

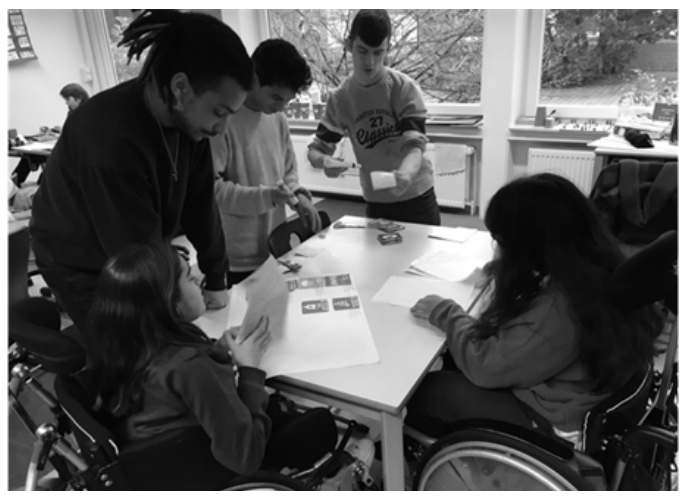

Abb. 1: Zusammenarbeit im Klassenzimmer der Mira Lobe Schule Hannover, Quelle: Stephanie Selke-Voigt 
Das menschliche Miteinander, das miteinander Leben und Lernen, egal welche Voraussetzungen man mitbringt, aus welcher Kultur oder welcher Familie man kommt, steht ebenfalls im Zentrum der pädagogischen Arbeit an der Mira Lobe Schule. Niemand wertet hier eine Schülerin oder einen Schüler aufgrund eines möglichen Stigmas. Es ist normal, verschieden zu sein. Behinderungen oder das Herkunftsland treten in den Hintergrund. In Klassenräten wird Demokratie gelebt. Das Schulsprecherteam für die Förder- und Oberschule besteht aus Jugendlichen beider Schulzweige.

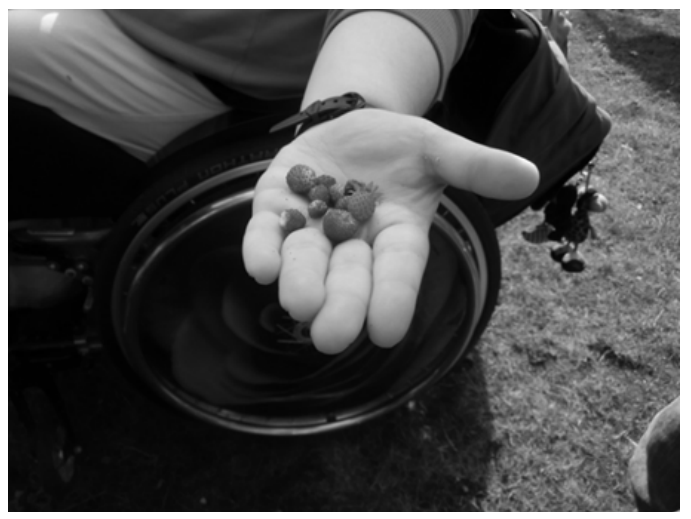

Abb. 2: Schülerinnen und Schüler ernten Obst an der Mira Lobe Schule Hannover, Quelle: Marcel Domeier

\section{Was fällt auf? Zusammenfassung der Interviews}

Viele Aspekte der 17 SDGs sind im Schulalltag noch gar nicht in Augenschein genommen oder bisher vernachlässigt worden. In der Vorbereitung auf die Interviews wurde deutlich, wie kompliziert die Ziele teilweise formuliert sind. Die Schülerinnen und Schüler des 7. Jahrgangs haben versucht, sie in eine ihnen verständliche Sprache zu übersetzen. Das war nicht immer leicht.

Die Interviews wurden sowohl im Förder- als auch im Oberschulbereich durchgeführt. Die Förderschulklassen arbeiten nur punktuell im inklusiven Setting. Der Unterricht in den Oberschulklassen hingegen ist durchgängig inklusiv. Interessant ist, dass die Interview-Antworten trotzdem kaum voneinander abweichen.

Alle Schülerinnen und Schüler machen sich Sorgen um die Erde. Viele verfolgen die aktuellen Nachrichten oder informieren sich in den sozialen Medien. Sie fragen sich, wie die Erde in einigen Jahren bzw. Jahrzehnten aussehen wird: „Ich mache mir Sorgen um die Tiere. Viele Tierarten sterben aus oder sind vom Aussterben bedroht." „Was passiert, wenn immer mehr Regenwald gerodet wird? Wie wird es den Menschen gehen?" Eine Frage, die immer wieder gestellt wurde: „Wann wird endlich die Politik auf die verheerenden Katastrophen und Veränderungen reagieren? Warum geschieht nichts oder nur wenig? Sollte es nicht viel strengere Gesetze geben?“. „Ich ärgere mich sehr, wenn ich so etwas höre, und frage mich, warum die Menschen auf der Erde nicht dafür sorgen können, dass so etwas nicht passiert.“ Oder: „Ich mache mir Gedanken, was in 10 Jahren sein wird." Es wird in den Interviews sehr deutlich, dass die Schülerinnen und Schüler das Thema ernst nehmen, dass es auch emotionale Aspekte für sie hat und dass es ihren Alltag begleitet.
Viele der Jugendlichen haben in ihrem direkten Umfeld und in den Familien angefangen umzudenken. Einige fahren weniger mit dem Auto, andere kompostieren selbst und bauen frisches Gemüse im eigenen Garten an. Viele sparen Wasser oder Strom. Sie versuchen, Plastik zu vermeiden und den Müll richtig zu sortieren, obwohl das selbst in einer Großstadt wie Hannover nicht immer einfach ist. Die Jugendlichen geben in den Interviews sehr viele ganz konkrete Hinweise, wie sie selbst handeln und was sie anderen raten. Es wird deutlich, dass sich die meisten Schülerinnen und Schüler eine schnellere, radikalere Änderung der jetzigen Situation wünschen. Ihnen gehen die Maßnahmen der Eltern oft noch nicht weit genug, obwohl Klimaschutz zu Hause durchaus ein Thema ist. Dennoch sollte es ihrer Meinung nach wesentlich weniger bis gar kein Fleisch zu essen geben. Auch der Kauf von Bio-Lebensmitteln ist den Jugendlichen wichtig. Plastikprodukte sollten gemieden werden, da es gute Alternativen aus Glas, Blech oder Naturprodukten gibt.

Warum es für alle Menschen in der Gesellschaft wichtig ist, sich mit nachhaltiger Entwicklung auszukennen? „Damit man schon vorher einschätzen kann, was man für Folgen verursacht, wenn man bestimmte Dinge entwickelt oder nutzt." Die Schule scheint nach Meinung der Schülerinnen und Schüler eine gute Botschafterin für Nachhaltigkeitsthemen zu sein. Sie geben an, dass viele Lehrerinnen und Lehrer wichtige Aspekte aufgreifen („Die Lehrer bringen uns vieles bei, was wir nicht wissen.") und Bezug auf aktuelle Ereignisse nehmen. Lehrkräfte können gute Vorbilder sein: „Viele Lehrer kommen immer mit dem Fahrrad zur Arbeit“. Es wurde jedoch auch mehrmals der Verdacht geäußert, dass im Unterricht zu viel Papier verbraucht würde. Die Eltern achteten zwar vielfach

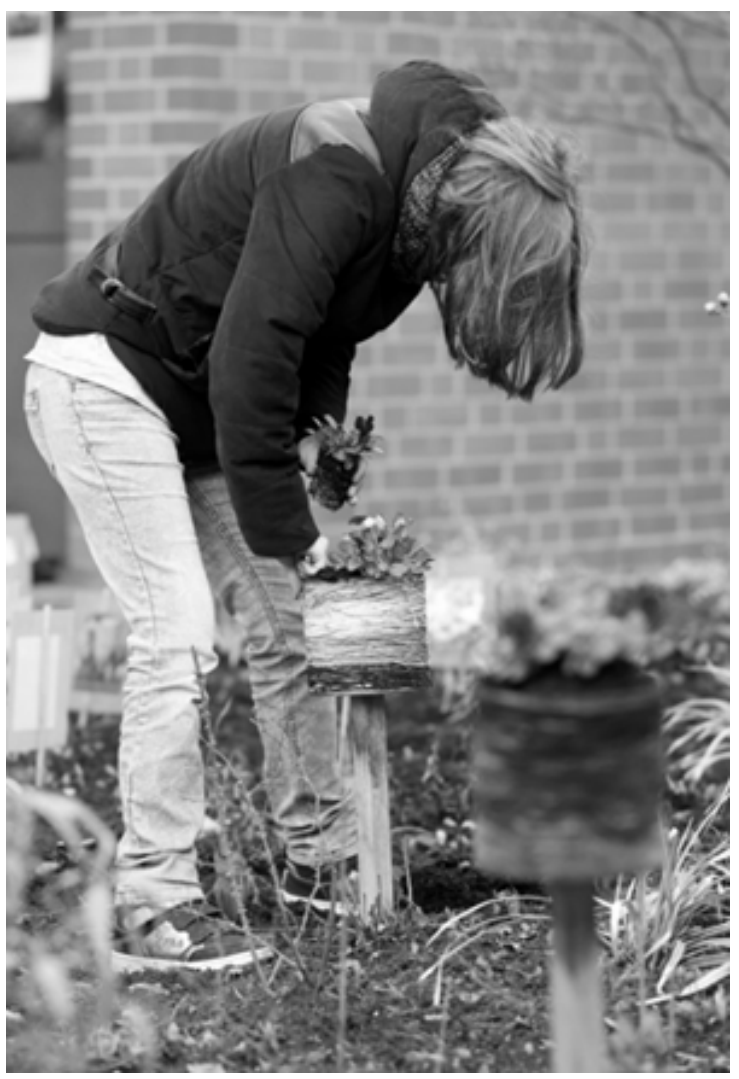

Abb. 3: Schülerinnen und Schüler gärtnern an der Mira Lobe Schule Hannover, Quelle: Marcel Domeier 
schon auf Nachhaltigkeit, würden aber durch das neu erworbene Wissen der Jugendlichen in ihrem Handeln bestärkt und motiviert, selbst etwas zu tun. Die in den Schulalltag integrierten Aufgaben wie das Müllsammeln auf dem Schulhof, das Wegbringen des Altpapiers oder das Pflegen der Gemüse- und Kräuterbeete sind für die meisten inzwischen selbstverständlich geworden. Spiele, Projekte, Internet-Recherchen und praktisches Tun helfen, sich im Unterricht noch intensiver mit den Sachverhalten auseinanderzusetzen.

Viele Schülerinnen und Schüler machen sich mittlerweile Gedanken darüber, was sie darüber hinaus unternehmen könnten, um das Thema noch weiter in den Fokus zu rücken. Eine Klasse hat gemeinsam bei Fridays for Future demonstriert. Aber auch der Austausch untereinander ist ihnen wichtig. Ideen und Tipps aus dem persönlichen Umfeld werden weitergegeben. Sie können einander von ihren Erfahrungen berichten und sich gegenseitig vieles erklären und unterstützen. Das Lernen in einem inklusiven System begünstigt ihrer Meinung nach diese Möglichkeiten. Weil alle ihre Stärken und Schwächen einsetzen, werden gemeinsam gesteckte Ziele erreicht. Jeder kann etwas Wichtiges dazulernen und somit etwas für die Umwelt tun.

„Heute schon an morgen denken.“

„Für später sorgen.“

„Die Welt retten.“
Diese Schlagwörter fielen im Kontext der Interviews. Der Unterricht zum Thema Nachhaltigkeit wird positiv gesehen. Auch jüngere Menschen müssten motiviert werden, etwas gegen den Klimawandel zu tun. Mehrere Schülerinnen und Schüler gaben an, dass sie es schwierig finden, alle Bedingungen einzuhalten, die zu einem nachhaltigeren Leben führen. „Wir haben gelernt, wie sehr wir durch unser Tun der Umwelt schaden."Aber während einige sagen: „Schwierig ist, alles einzuhalten.“, schätzen andere ein: „Das Thema war einfach zu lernen.“. Dennoch müsse man im privaten Bereich beginnen, sich mit den Hintergründen auseinandersetzen und nicht aufgeben, damit sich mittelfristig auch im Bereich der Industrie und in der Politik etwas bewegt. Alle sind sich einig, dass sie etwas für die Umwelt tun wollen. Jeder könne bei sich selbst damit beginnen. Sich aber in einer Gemeinschaft zusammen auf den Weg zu machen, sich Mut zuzusprechen und Sachverhalte zu diskutieren, verstärke den Effekt. Während der Interviews wurde dies ganz konkret ausgesprochen: „Wir sind der Meinung, dass alle Kinder und Jugendlichen gemeinsam am besten lernen können.“

Die Schülerinnen und Schüler der Mira Lobe Schule sind gemeinsam auf dem Weg. Vieles muss noch wachsen, vieles muss noch selbstverständlicher werden. Ein Grundverständnis füreinander ist jedoch bereits angelegt. Hier wird Inklusion greifbar und konkret.

Stephanie Selke-Voigt, Stellvertretende Schulleiterin, Mira Lobe Schule doi.org/10.31244/zep.2020.01.06 